\title{
Regulating the surface passivation and residual strain in pure tin perovskite films
}

Manman $\mathrm{Hu}^{1}$, Riming $\mathrm{Nie}^{1}$, Hyeonwoo $\mathrm{Kim}^{1}$, Jianchang $\mathrm{Wu}^{2}$, Shanshan $\mathrm{Chen}^{3}$, Byung-wook Park $^{1}$, Gwisu Kim ${ }^{1}$, Hyoung-Woo Kwon ${ }^{1}$, Sang Il Seok ${ }^{1, *}$.

${ }^{1}$ Department of Energy and Chemical Engineering, Ulsan National Institute of Science and Technology, 50 UNIST-gil, Eonyang-eup, Ulju-gun, Ulsan 44919, Korea.

${ }^{2}$ Institute of Materials for Electronics and Energy Technology (i-MEET), Department of Materials

Science and Engineering, Friedrich-Alexander-Universität, Erlangen-Nürnberg (FAU), Martensstraße 7, 91058 Erlangen, Germany.

${ }^{3}$ School of Energy \& Power Engineering, MOE Key Laboratory of Low-Grade Energy Utilization Technologies and Systems, CQU-NUS Renewable Energy Materials \& Devices Joint Laboratory, Chongqing University, Chongqing 400044, P. R. China

*Corresponding author. Email: seoksi@unist.ac.kr 


\section{Methods}

\section{Materials}

All chemicals were purchased from commercial suppliers and were used as received unless specified otherwise. Tin iodide $\left(\mathrm{SnI}_{2}, 99.99 \%\right)$, cesium iodide $(\mathrm{CsI})$, and tin fluoride $\left(\mathrm{SnF}_{2}, 99 \%\right)$ were purchased from Sigma-Aldrich. Poly(triarylamine) (PTAA, $\left.M_{n}=17500\right)$ was purchased from the EM Index. 4-isopropyl-4'-methyldiphenyliodoniumtetrakis(pentafluorophenyl)borate (DPI-TPFB) was purchased from TCI.

\section{Formanidinium iodide (FAI) synthesis}

Formanidinium iodide (FAI) was synthesized by mixing $20 \mathrm{mg}$ of formamidine acetate salt $(99 \%$, Alfa Aesar) with $30 \mathrm{~mL}$ of hydroiodic acid (HI, $57 \mathrm{wt} \%$ in water, Aldrich ) in a 250-mL roundbottom flask at $60{ }^{\circ} \mathrm{C}$ under reduced pressure for $60 \mathrm{~min}$. The products were first dissolved in 200 $\mathrm{ml}$ ethanol and then recrystallized overnight from diethyl ether in a refrigerator. Finally, the FAI powder was dried in a vacuum oven for at least half a day.

\section{2-Thiophenemethylammonium iodide (ThMAI) synthesis}

In this procedure, $7 \mathrm{~mL}$ of hydroiodic acid (HI, $57 \mathrm{wt} \%$ in water, Sigma-Aldrich) was allowed to react with $5 \mathrm{~mL}$ of thiophenemethylamine $(98 \%, \mathrm{TCl})$ in $15 \mathrm{~mL}$ ethanol at $0{ }^{\circ} \mathrm{C}$ for $2 \mathrm{~h}$ by stirring. The product was first dissolved in ethanol, then recrystallized from diethyl ether, and finally, it was dried at room temperature in a vacuum oven overnight.

\section{Device fabrication}

PSC devices were fabricated on pre-cleaned patterned fluorine-doped $\mathrm{SnO}_{2}$ (FTO, Pilkington, 
TEC $8,8 \Omega / \mathrm{cm}^{2}$ ) glass substrates. The substrates were pre-cleaned in an ultrasonic bath with detergent, water, acetone, isopropanol, and ethanol for $30 \mathrm{~min}$ each, followed by ultraviolet ozone treatment to remove the organic residuals.

Hole-blocking $\mathrm{TiO}_{2}\left(\mathrm{bl}_{-}-\mathrm{TiO}_{2}\right)$ was deposited onto the $\mathrm{FTO} /$ glass substrate by spray pyrolysis deposition of a $20 \mathrm{mM}$ titanium diisopropoxide bis(acetylacetonate ) (75 wt\%, Sigma-Aldrich) solution at $450{ }^{\circ} \mathrm{C}$. Mesoporous $\mathrm{TiO}_{2}\left(\mathrm{mp}-\mathrm{TiO}_{2}\right)$ was spin-coated at $1500 \mathrm{rpm}$ for $50 \mathrm{~s}$ on the bl$\mathrm{TiO}_{2} / \mathrm{FTO}$ substrate using a $\mathrm{TiO}_{2}$ paste in 2-methoxyethanol (2-ME, 99.8\%, Sigma-Aldrich) and terpinol (90\%, Sigma-Aldrich) in a weight ratio of 3.5:1. The films were annealed at $500{ }^{\circ} \mathrm{C}$ for $60 \mathrm{~min}$. The perovskite layer was deposited using a one-step spin-coating method. Then, $409.8 \mathrm{mg}$ $\mathrm{SnI}_{2}, 154.8 \mathrm{mg}$ FAI, $13 \mathrm{mg} \mathrm{CsI}$, and $24 \mathrm{mg} \mathrm{SnF}_{2}$, were dissolved in a mixed solvent of $700 \mu \mathrm{L} N$, $N$-dimethylformamide (DMF) and $300 \mu \mathrm{L}$ dimethyl sulfoxide (DMSO). The precursors were deposited on the mesoporous layers at $3000 \mathrm{rpm}$ for $40 \mathrm{~s}$. The film, which became dark brown during spin-coating, was dried on a hot plate at $120^{\circ} \mathrm{C}$ for $10 \mathrm{~min}$. For ThMAI treatment, the ThMAI solutions were dissolved in isopropanol (IPA) at different concentrations ranging from 0 to $4 \mathrm{mg}$ and spin-coated onto the perovskite surface at a speed of $5000 \mathrm{rpm}$, followed by annealing at $100{ }^{\circ} \mathrm{C}$ for $10 \mathrm{~min}$. Then, a solution of the HTM consisting of $20 \mathrm{mg}$ of PTAA and $2.25 \mathrm{mg}$ of DPI-TPFB in $1 \mathrm{~mL}$ chlorobenzene was spin-coated onto the top of the perovskite layer at 1500 rpm for $30 \mathrm{~s}$, followed by thermal annealing for $5 \mathrm{~min}$ at $70{ }^{\circ} \mathrm{C}$; this entire process was conducted in the dark. All procedures were performed in a glovebox with oxygen and moisture levels of approximately $40 \mathrm{ppm}$. To complete the device, a 60 -nm-thick Au electrode was thermally evaporated on top of the hole-transporting layer using a shadow mask. A $0.094 \mathrm{~cm}^{2}$ non-reflective metal mask was used for measurement purposes to accurately define the active area of the solar cells. 


\section{Characterization}

The $J-V$ curves were measured using a Keithley 2420 source meter and a solar simulator (Newport, Oriel Class A, 91195A) producing $100 \mathrm{~mW} \cdot \mathrm{cm}^{-2}$ illumination (AM $1.5 \mathrm{G}$ ). The unencapsulated devices were measured both in reverse [forward bias $(1.2 \mathrm{~V}) \rightarrow$ short circuit $(0 \mathrm{~V})$ ] or forward [short circuit $(0 \mathrm{~V}) \rightarrow$ forward bias $(1.2 \mathrm{~V})$ ] scan modes. The step voltage and delay time were fixed at $0.1 \mathrm{~V}$ and $40 \mathrm{~ms}$, respectively. External quantum efficiency (EQE) was measured using an internal quantum efficiency system (Oriel, IQE 200B) under irradiation by a $100 \mathrm{~W}$ Xenon lamp. Film morphologies were measured using a scanning electron microscope (Cold FE-SEM SU-8220). The SCLC characteristics and dark $J-V$ curves were measured using a Keithley 2636 B source meter. GIXRD measurements were performed on Rigaku D8 Discovery. GIWAXD measurements were conducted at the PLS-II 6D UNIST-PAL beamline of the Pohang Accelerator Laboratory. During scanning, the samples were exposed to X-rays $(\lambda=0.62382 \AA)$ at varying angles of incidence $\left(0.117^{\circ}, 0.145^{\circ}, 0.198^{\circ}\right.$, and $\left.0.3^{\circ}\right)$ for $30 \mathrm{~s}$. The XRD patterns were obtained using a Rigaku D/MAX-2500 system with $\mathrm{Cu}-K_{\alpha}$ radiation $(\lambda=1.5405 \AA)$ as the $\mathrm{X}$-ray source. Both UPS and XPS analyses were performed using Thermo Fisher Scientific (ESCALAB 250XI). EIS was performed using a Metrohm Autolab instrument (AUT302N). The optical properties of the films were measured using UV-Vis spectroscopy (Shimadzu UV-2600). Steady-state PL and TRPL spectra were recorded using a commercial time-correlated single photon counting (TCSPC) setup (FluoTime 300, PicoQuant GmbH) equipped with a PMA-C-192-M detector and highresolution excitation monochromators. The composition of the films was analyzed using secondary ion mass spectrometry (TOF-SIMS5, UNIST Central Research Facilities) with a $\mathrm{Bi}^{3+}$ primary beam $(25 \mathrm{keV}, 0.43 \mathrm{pA})$ and an $\mathrm{O}_{2}$ sputter beam $(500 \mathrm{keV}, 85 \mathrm{nA})$. 

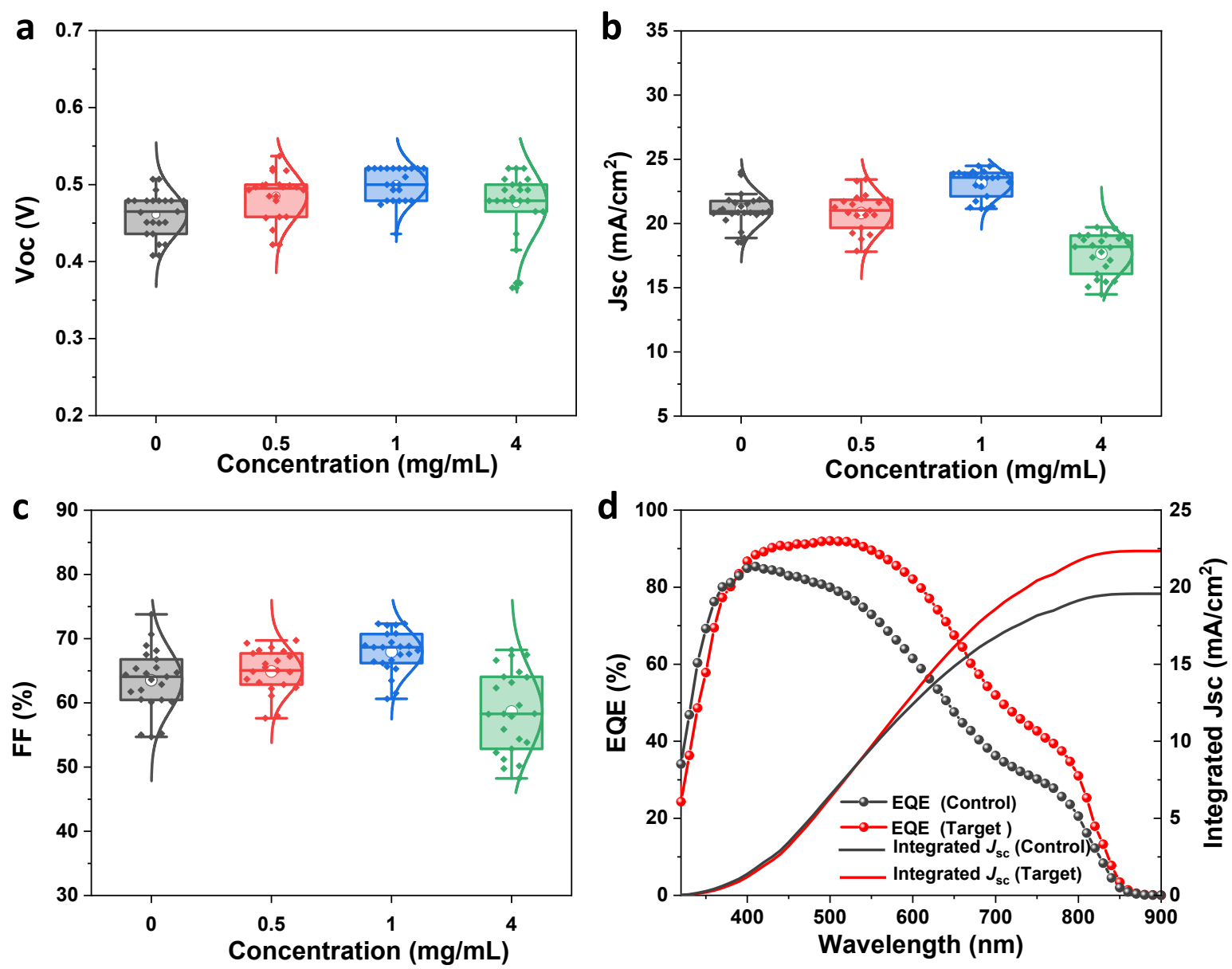

Supplementary Figure 1 (a) Open-circuit voltage $\left(V_{o c}\right)$. (b) Short-circuit current density $\left(J_{s c}\right)$. (c) Fill factor $(F F)$. The upper and lower error bars represent the maximum and minimum values respectively, and the mid-line in each box represents the median value. The top and the bottom of box represent the upper quartile ( 75 th percentile) and lower quartile (25th percentile), respectively, and box height represents the interquartile range. (d) EQE spectrum and the relevant integrated JSC.

Table S1 Solar cell performance parameters determined from J-V curves and stabilized power output measurements

\begin{tabular}{l|llll}
\hline Device & PCE $(\%)$ & Voc $(\mathrm{V})$ & Jsc $\left(\mathrm{mA} / \mathrm{cm}^{2}\right)$ & FF (\%) \\
\hline $\begin{array}{l}\text { Control } \\
\text { Average } \\
\text { Target }\end{array}$ & $6.17 \pm 0.66$ & $0.461 \pm 0.029$ & $21.12 \pm 1.27$ & $63.51 \pm 4.82$ \\
Average & $7.86 \pm 0.99$ & $\mathbf{0 . 5 0} \pm 0.021$ & $23.15 \pm 0.79$ & $67.95 \pm 2.78$ \\
\hline
\end{tabular}


Average device characteristics with standard deviation were obtained on the basis of 24 cells for each set. 

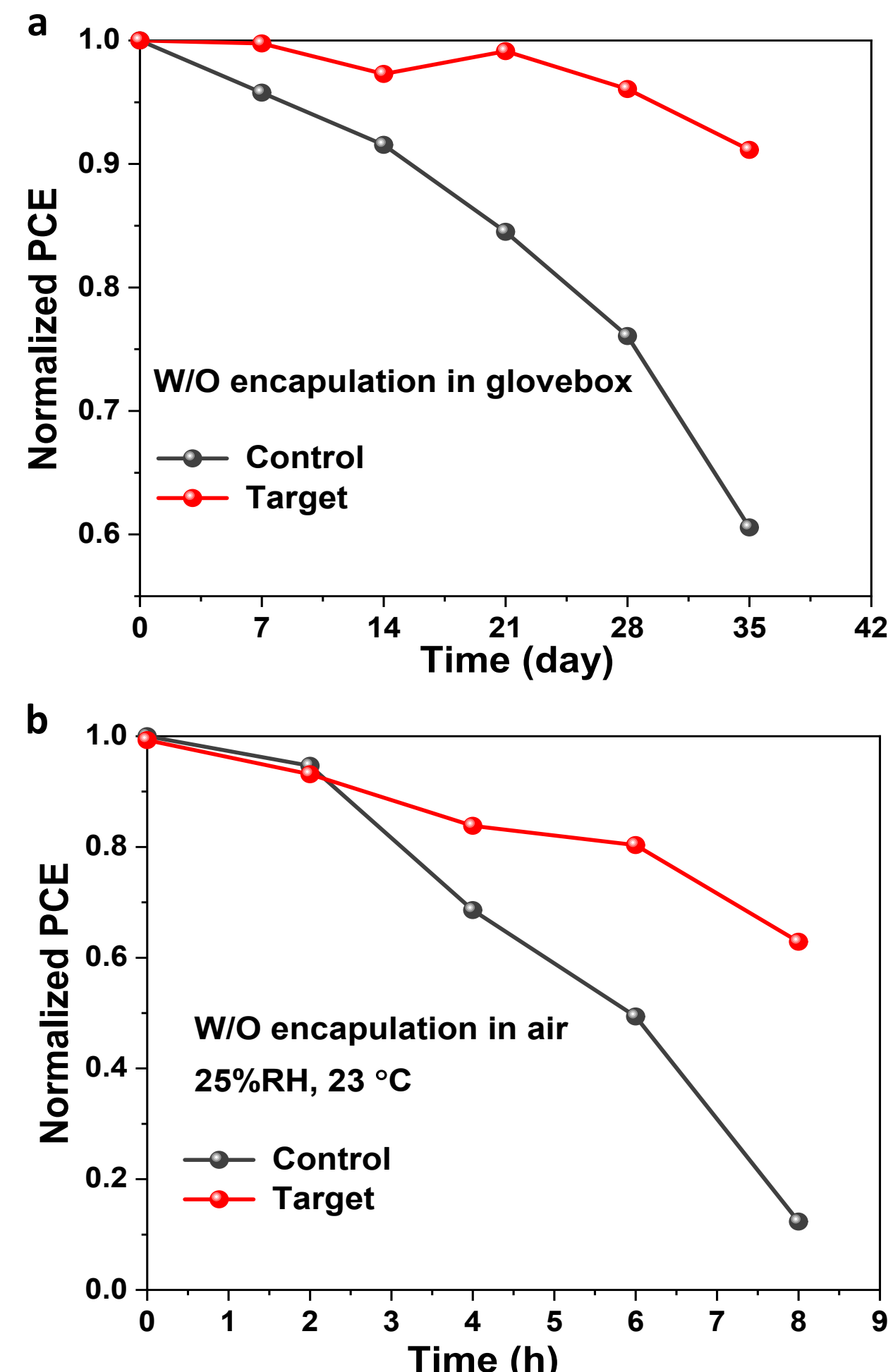

Supplementary Figure 2 The stability of the control and the target devices, stored in glove box and in air, over time. 
Table S2 tDOS values calculated from the tDOS profiles.

\begin{tabular}{llll}
\hline & $\begin{array}{l}\text { Shallow trap }\left(\mathbf{c m}^{-3}\right) \\
\text { (percentage) }\end{array}$ & $\begin{array}{l}\text { Deep trap }\left(\mathrm{cm}^{-3}\right) \\
\text { (percentage) }\end{array}$ & $\begin{array}{l}\text { Total }\left(\mathbf{c m}^{-3}\right) \\
\text { (percentage) }\end{array}$ \\
\hline Control & $2.33 \times 10^{17}(3.03 \%)$ & $7.45 \times 10^{18}(96.97 \%)$ & $7.68 \times 10^{18}(100 \%)$ \\
\hline Target & $7.15 \times 10^{17}(13.91 \%)$ & $4.43 \times 10^{18}(86.09 \%)$ & $5.14 \times 10^{18}(100 \%)$ \\
\hline
\end{tabular}



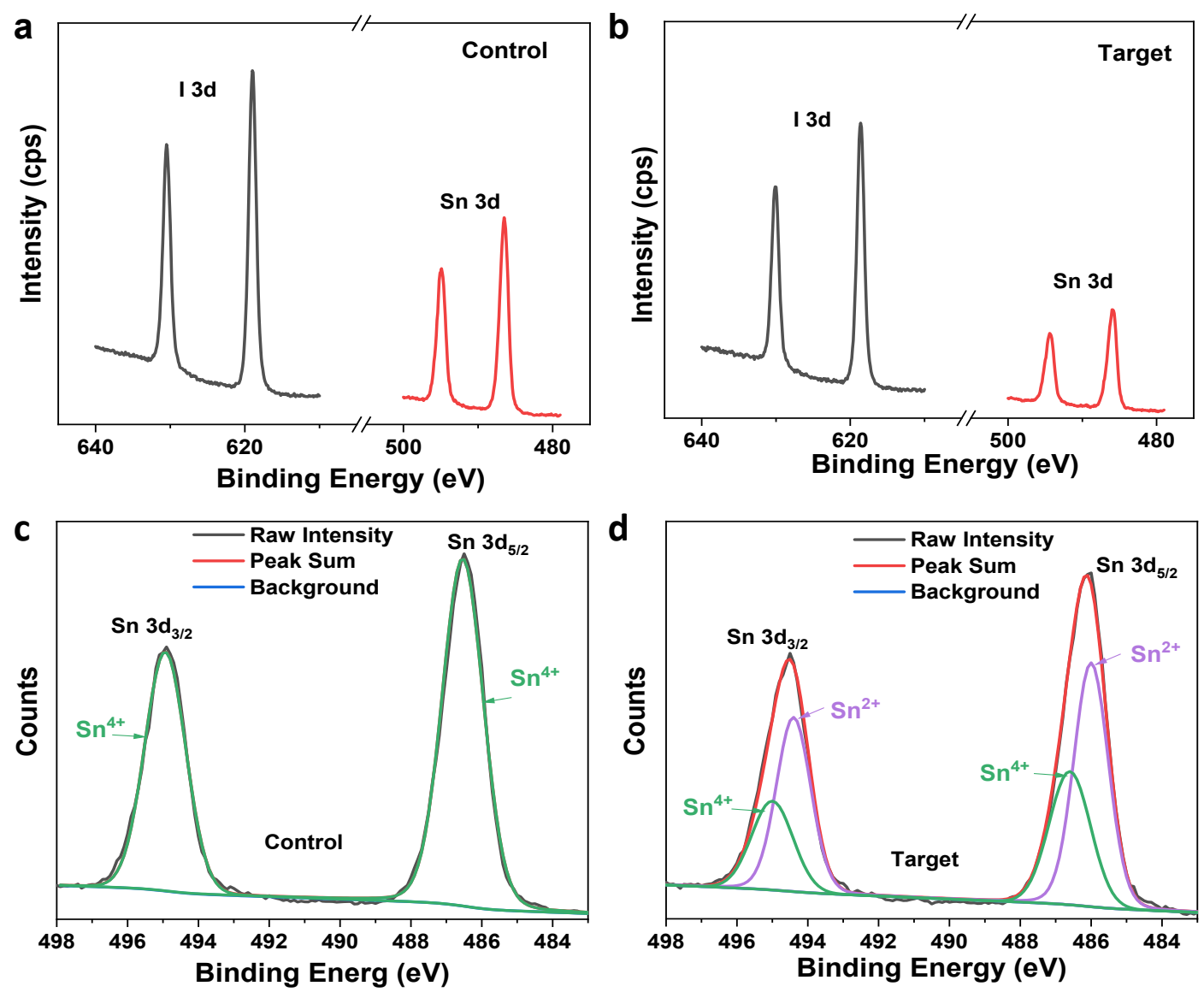

Supplementary Figure $3 \mathrm{Sn} 3 \mathrm{~d}$ and I $3 \mathrm{~d}$ core energy level spectra from $\mathrm{Cs}_{0.1} \mathrm{FA}_{0.9} \mathrm{SnI}_{3}$ without (a) and with (b) ThMAI coating (target). It can be estimated that ratio of $\mathrm{Sn} / \mathrm{I}$ are 1: 1.39 and 1: 1.52 for perovskite film without and with ThMAI coating, respectively, indicating that the iodine amount at the surface has been significantly increased, which could reduce the iodine vacancies defects on the surface. (c) Control and (d) ThMAI (1mg in $1 \mathrm{~mL}$ IPA) show distributions of $\mathrm{Sn}^{2+}$ and $\mathrm{Sn}^{4+}$ in their surface states. 
a
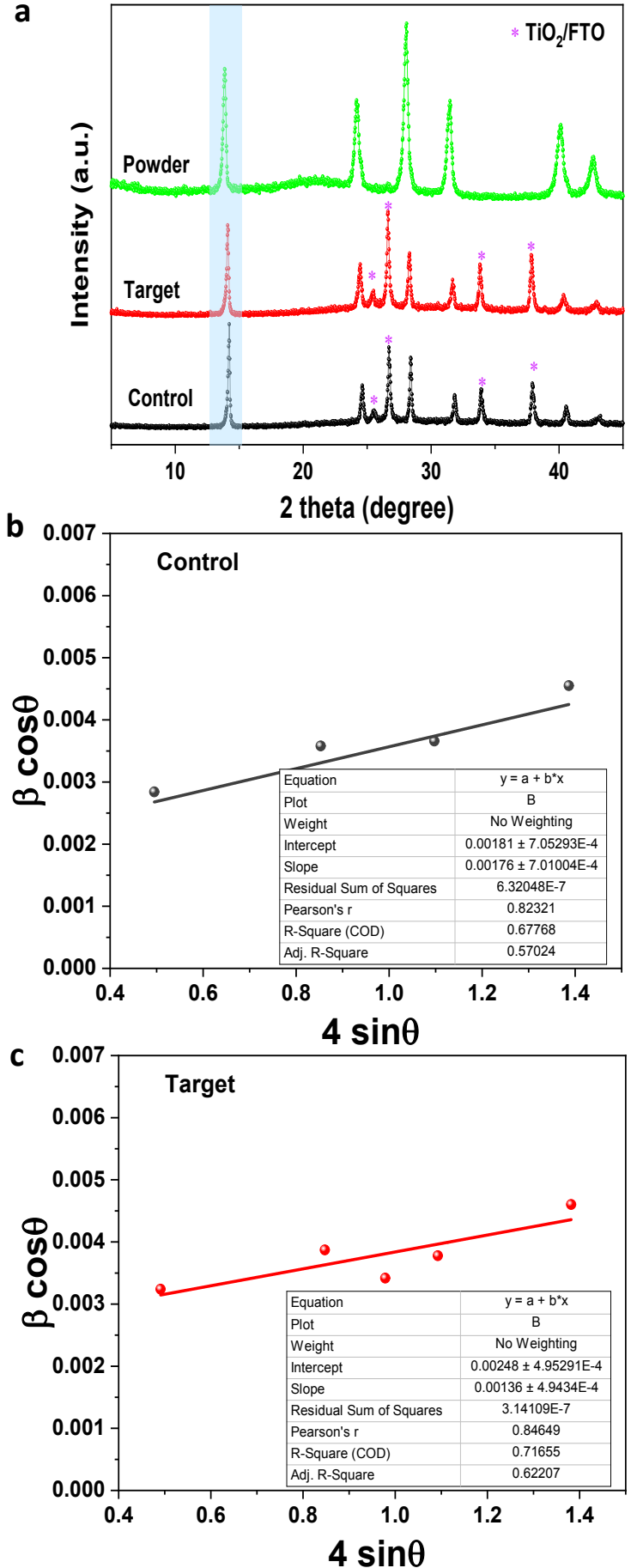

Supplementary Figure 4 (a) Out-of-plane XRD patterns of powder, target, and control film. Williamson-Hall plots :(b) control and (c) target of $\mathrm{Cs}_{0.1} \mathrm{FA}_{0.9} \mathrm{SnI}_{3}$ perovskite films. 
In the XRD data, the broadening $\left(\beta_{\mathrm{T}}\right)$ of the peaks is due to the combined effect of the crystallite size $\left(\beta_{\mathrm{D}}\right)$ and micro strain $\left(\beta_{\varepsilon}\right)$, i.e.,

Total broadening $=$ Broadening arising from crystallite size + Broadening arising from strain

$$
\beta_{T}=\beta_{D}+\beta_{\varepsilon} \quad----(1)
$$

Where $\beta_{\mathrm{T}}$ is the total broadening, $\beta_{\mathrm{D}}$ is broadening arising from crystallite size and $\beta_{\varepsilon}$ is the broadening arising from strain.

From the Scherer equation, we know that

$$
\beta_{D}=\frac{K \lambda}{D \cos \theta}-----(2)
$$

where $\beta_{\mathrm{D}}$ is the FWHM (i.e. broadening of the peak) in radians, $\mathrm{K}=0.9$ is the shape factor, $\lambda=$ $0.15406 \mathrm{~nm}$ is the wavelength of X-ray source, $\mathrm{D}$ is the crystallites size and $\theta$ is the peak position in radians.

Similarly, the XRD peak broadening arising from micro strain is given by,

$$
\beta_{\varepsilon}=4 \varepsilon \tan \theta----(3)
$$

where $\beta_{\varepsilon}$ is broadening arising from strain, $\varepsilon$ is the strain and $\theta$ is the peak position in radians.

Putting equation (2) and (3) in equation (1), we obtain

$$
\beta_{T} \cos \theta=\varepsilon(4 \sin \theta)+\frac{K \lambda}{D}----(4)
$$

Equation (4) represents a straight line, in which $\varepsilon$ is the gradient (slope) of the line and $\frac{K \lambda}{D}$ is the $y-$ intercept.

Considering the standard equation of a straight line,

$$
\mathrm{y}=\mathrm{mx}+\mathrm{c}----(5)
$$

where " $\mathrm{m}$ " is the slope of line and " $\mathrm{c}$ " is the $\mathrm{y}$-intercept, and comparing equation (4) and (5), we obtain,

$$
\begin{array}{ll}
\mathrm{y}=\beta_{T} \cos \theta & ----(i) \\
\mathrm{m}=\varepsilon & ----(\mathrm{ii})
\end{array}
$$




$$
\begin{aligned}
& \mathrm{x}=4 \sin \theta \quad--(i i i) \\
& \mathrm{c}=\frac{K \lambda}{D} \quad---(i v)
\end{aligned}
$$

Then, $(4 \sin \theta)$ is plotted on the $\mathrm{x}$-axis and $\left(\beta_{T} \cos \theta\right)$ on the $\mathrm{y}$-axis, and value of " $m$ " which represent gradient (slope) of the line gives the strain " $\varepsilon$ ". 

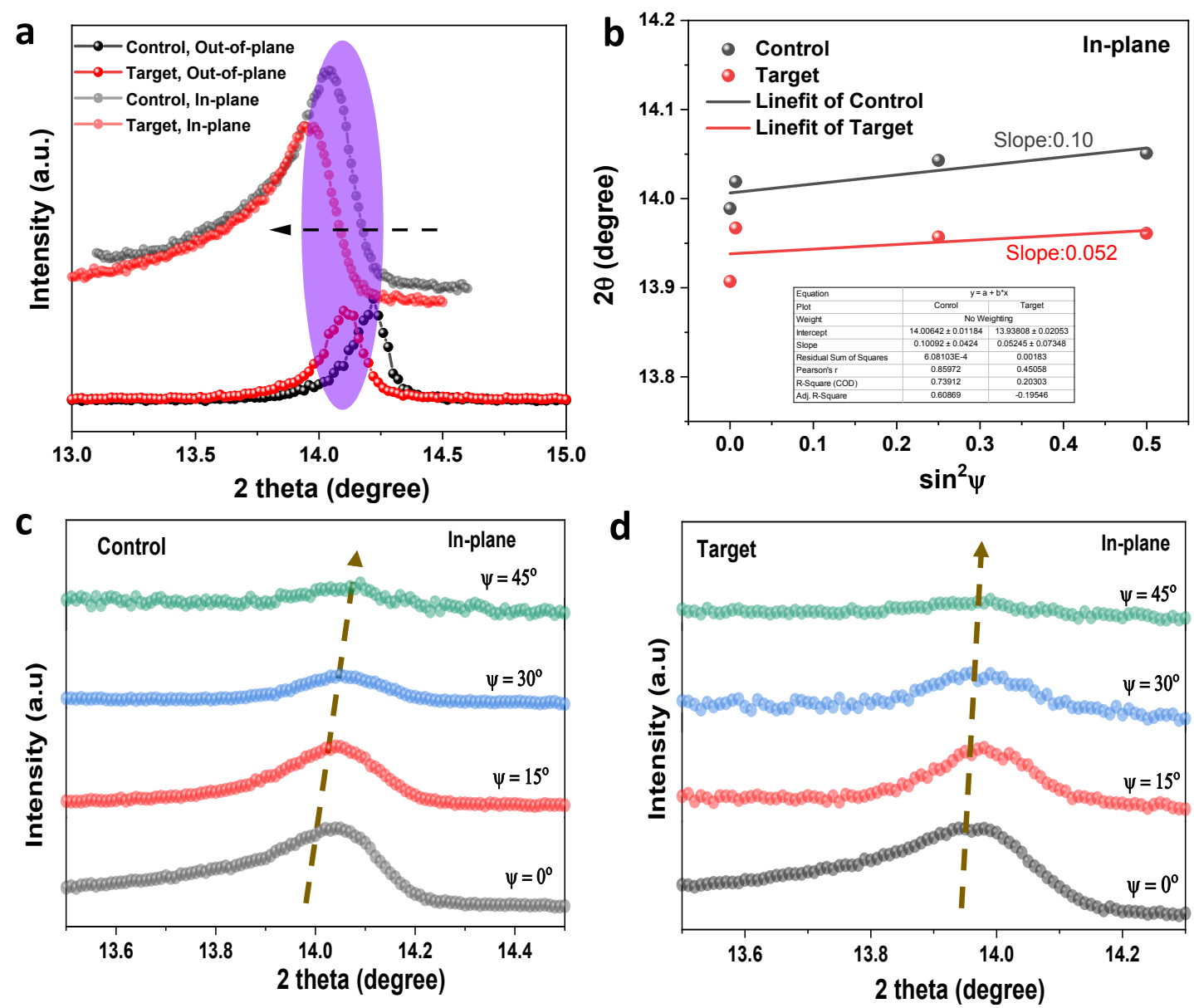

Supplementary Figure 5 (a) The GIXRD (in-plane) and normal XRD (out-of-plane) spectra of a (100) peak of control and target samples. (b) Compressive strain distribution of (100) crystal peak as a function of $\sin ^{2} \psi$. (c) and (d) GIXRD spectrum at different tilt angles, where the incident angle $\alpha$ is $0.5^{\circ}$. 

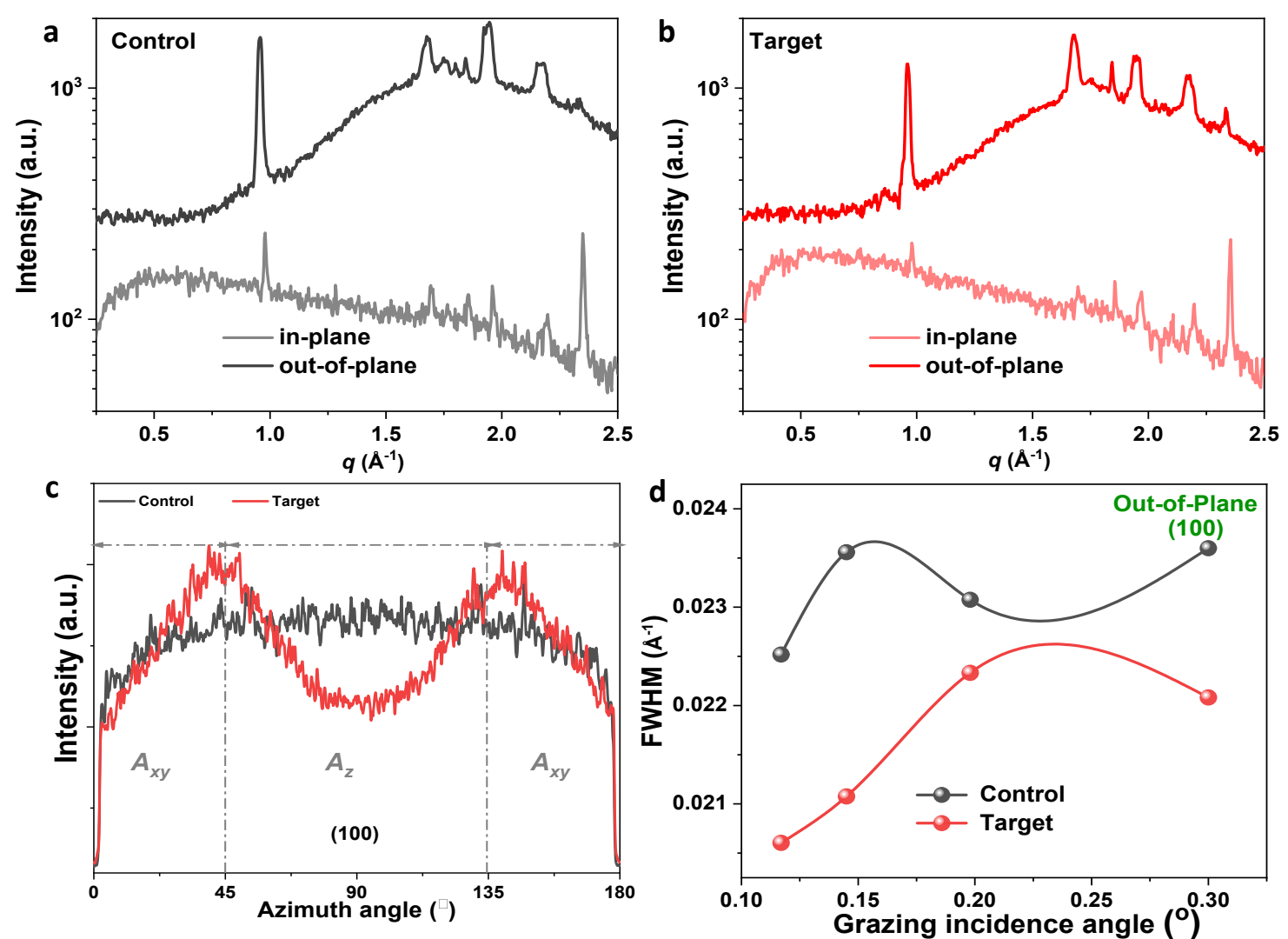

Supplementary Figure 6 (a) and (b) The (100) in-plane and out-of-plane line cuts of GIWAXD images. (c) Pole figure plots from (100) lamellar diffraction in parentheses are the ratios of integrated $0^{\circ}-45^{\circ}$ and $135^{\circ}-180^{\circ}(A x y)$ to $45^{\circ}-135^{\circ}(A z)$. (d) FWHM values obtained from (100) plane as a function of incident angle $\alpha$. 